\title{
Evaluation of Cervical Posture Following Rapid Maxillary Expansion: A Review of Literature
}

\author{
Eleonora Ortu ${ }^{1, *}$, Davide Pietropaoli ${ }^{1}$, Maurizio Ortu ${ }^{2}$, Mario Giannoni ${ }^{3}$ and Annalisa Monaco ${ }^{1}$ \\ ${ }^{l}$ Department of Life, Health and Environmental Sciences; Division of Gnatology; Dental Clinic; University of L'Aquila; \\ Via Vetoio 67100, L'Aquila, Italy \\ ${ }^{2}$ Otolaryngology and Audiology; Department of Life, Health and Environmental Sciences; Dental Clinic; University of \\ L'Aquila; L'Aquila, Italy \\ ${ }^{3}$ Department of Life, Health and Environmental Sciences; Dental Clinic; University of L'Aquila; L'Aquila; Italy
}

\begin{abstract}
Objectives: The aim of this article is to elucidate the state of the art about how rapid maxillary expansion (RME) produces changes in natural head position (NHP) in terms of cephalometric angles or inclinometer data during the growth period, according to the available literature.

Methods: Electronic search was done from January 2002 to June 2012 on PubMed, Cochrane and Scopus databases; in addition manual search was conducted as well. According to keywords, eleven papers were eligible for our purpose, but definitely six papers were selected in agreement with the inclusion/exclusion criteria.

Conclusions: The current literature suggests that the potential relationship between RME and NHP is still unclear. In fact, although the change in the position of the head after the rapid palatal expansion is evident clinically, current orthodontic literature does not provide conclusive evidence about the nature of this relationship.
\end{abstract}

Keywords: Cervical posture, children, natural head position (nhp), rapid maxillary expansion, rapid palatal expansion.

\section{INTRODUCTION}

Rapid Maxillary Expansion (RME) - or Rapid Palatal Expansion (RPE) - was introduced in 1860s by Angell for the treatment of maxillary constriction and consisted of a shaft with tubular nuts that was rotated using a wrench made from a dime [1]. To date, new technologically advanced tools have been introduced, but the final aim remains the same. Recent literature suggests that RME is the most predictable tool for the correction of the posterior cross bite, due to deficiency of maxillary width, providing to expand the transverse dimension of the palate and maxillary dental arch [2]. RME produces the separation of the two maxillary halves using an orthopaedic approach introducing lateral forces on horizontal maxillary processes and a medial force, produced by a screw activated twice a day [3]. When the mid-palatal suture is kept expanded for a retention period, the space obtained by separating the two halves of maxilla is replaced by newly formed bone [4]. Several Authors report changes in facial morphology and head posture caused by RME [5]. According to these reports, recent literature suggests modification on Natural Head Position (NHP) during

*Address correspondence to this author at the Department of Life, Health and Environmental Sciences; Division of Gnatology; Dental Clinic; University of L'Aquila; Via Vetoio 67100, L'Aquila; Italy;

Tel: +390862 434973; E-mail: eleortu@gmail.com and after RME application. Investigating the possible impact of RME in terms of change in NHP is a great and actual topic that needs to be deepened, because more aspects remain unclear. At today, the gold standard for studying NHP changes is the cephalometric analysis, but, due to the lack of specific standardized protocols to this aim, it leads to inability to compare results of different papers, and more powerful systematic or meta-analytic considerations couldn't be done. Different ways of evaluation of NHP do not allow a systematic review of literature or meta-analysis.

However, the influence of RME in NHP can be analyzed with reference to objective measurements from studies using comparable cephalometric analyses or inclinometer data.

\section{METHODS}

\section{Research Strategy}

The following electronic databases were selected for search from January 2002 to June 2012: PubMed, Cochrane and Scopus. The database queries were performed independently by two reviewers (EO and DP) according to key words listed in Table 1. Disagreement regarding inclusion was resolved by discussion. To avoid inappropriate exclusions, adjectives, nouns, plural and singular forms of all terms were used. No language restriction was applied. 
Table 1. Electronic databases used and search strategy.

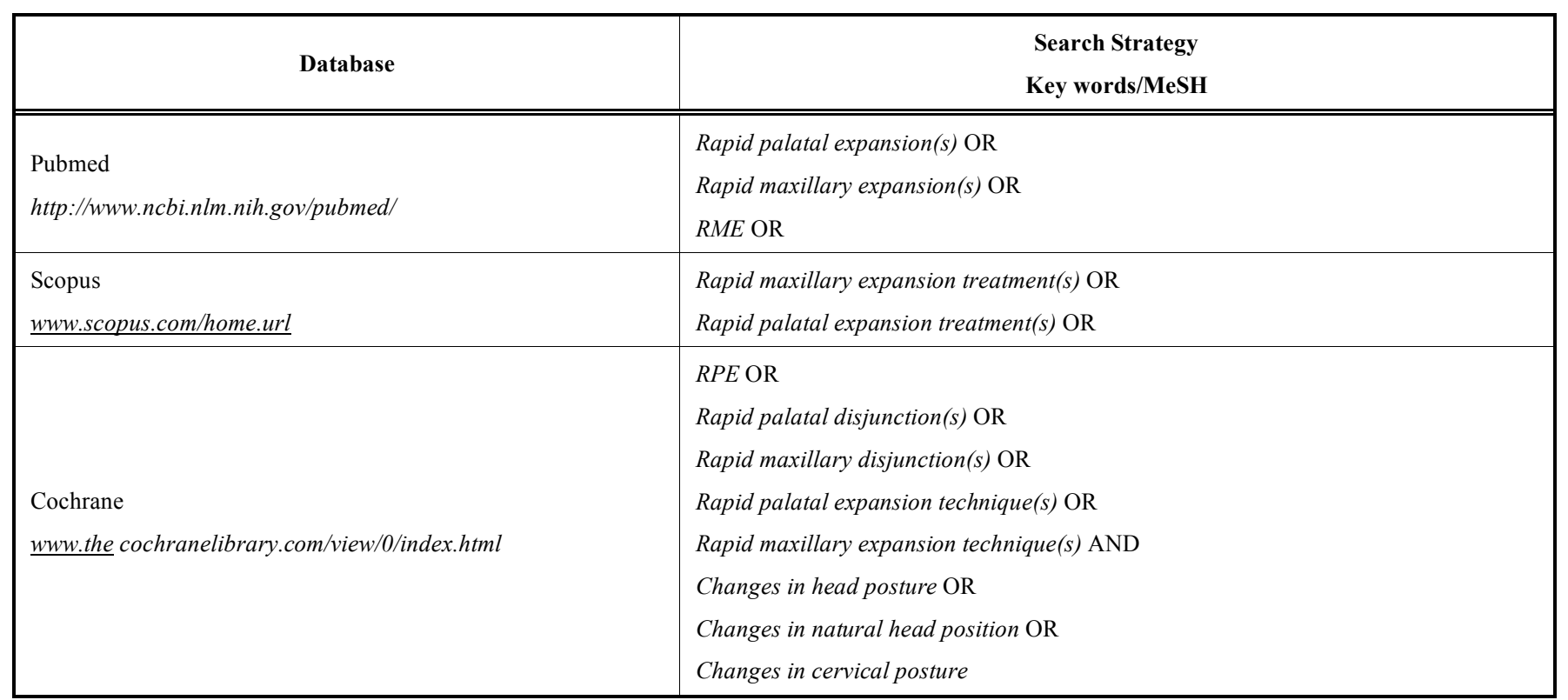

In addition, a manual search was performed in the following journals: European Journal of Orthodontics, American Journal of Orthodontics and Dentofacial Orthopedics, Angle Orthodontist and Australian Orthodontic Journal from January 2002 to June 2012.

The search resulted in a total of 11 articles and, subsequently, a final eligibility screening was conducted to verify the agreement with the inclusion and exclusion criteria listed below.

\section{Inclusion Criteria}

- $\quad$ Randomized controlled trials (RCTs) and historical controls for clinical trials (HCTs) [6];

- Follow-up of at least 6 months after RME/RPE therapy;

- $\quad$ Subjects during growth period $(<16$ years);

\section{Exclusion Criteria}

- Studies involving patients with systemic diseases, psychosocial impairment, craniofacial abnormalities, or skeletal open bite;

- Absence of complete data from the beginning of therapy to the end of the follow- up $[7,8]$;

- Studies without the use of lateral cervical radiography and inclinometer to evaluate the effects and changes of NHP after RME RPE [9-11];

- No data for the evaluation of inclusion /exclusion criteria.

A total of six articles were included according to both inclusion and exclusion criteria. These are summarized in Table 2 with notations regarding Authors, year of publication, sample size, treatments and cephalometric index or inclinometer index.

\section{RESULTS}

Six studies were identified for the aim of our paper. In two studies, inclinometer was used for the evaluation of outcomes $[12,16]$; in the others, cephalometric indexes were used as described in Table 2 and in the Figs. (1-3) [13-15, 17]. The inclinometer and a portable data logger were used to collect the dynamic head posture data. The inclinometer were placed on the eyeglesses' arms. The right sensor was positioned parallel to the sagittal plane to determine changes in pitch and the left sensor was positioned vertical to the sagittal plane to determine changes in roll (transversal roll and sagittal pitch). Although an index-matched analysis could not be performed, due to the use of different indexes between studies, an evaluation by comparable measurements was conducted.

Yagci et al. [12] studied the changes in NHP after RME in 38 subjects. The treatment and the control groups consisted of 23 and 15 patients, respectively. The test subjects underwent RME treatment using full cap acrylic device. The midline expansion screw was activated twice a day for the first week and once a day thereafter, until the desired change in the transverse dimension was achieved. The appliance was left in place for one month after the active expansion period, and then it was used as a removable retention tool. An inclinometer and a portable data logger were used to collect the NHP data. The NHP recordings were made at the initial stage of appliance placement and at the end of the RME therapy, both in the treatment and in the control groups (mean observation period: 7-8 months). Subjects in the control group did not undergo any type of treatment during the observation period. The mean difference between initial and final NHP was $0.31^{\circ}$ and this difference was not statistically significant.

Tecco et al. [13] studied the long-term effects of RPE on nasopharyngeal airway size, head posture, and cervical 
Table 2. Characteristics of the selected studies. Cephalometric indexes - CVT: the upper part of the cervical spine. EVT: the lower part of the cervical spine. OPT: odontoid line. SN: anterior cranial base. PP: palatal plane. MP: mandibular plane. CVT/EVT: cervical lordosis angle. OPT/Ver: odontoid angle. EVT/Ver: lower cervical column angle. CVT/Ver: upper cervical column angle. SN/Ver: the downward opening angle between the SN and true vertical lines. PP/Ver: the downward opening angle between the palatal and true vertical lines. MP/Ver: the downward opening angle between the mandibular and true vertical lines. SN/OPT: the downward opening angle between OPT and SN lines. SN/CVT: the downward opening angle between CVT and SN lines. PP/OPT: the downward opening angle between OPT and palatal lines. PP/CVT: the downward opening angle between CVT and palatal lines. MP/OPT: the downward opening angle between OPT and mandibular lines. MP/CVT: the downward opening angle between CVT and mandibular lines. OPT/NSL: the angle formed by the line tangent to the odontoid process (CV2tp) through cv2ip (the most inferior and posterior point on the corpus of the second cervical vertebra), and the nasion-sella line. CVT/NSL: the angle formed by the line tangent to the odontoid process through cv4ip (the most inferior and posterior point on the fourth cervical vertebra), and the nasion-sella line. OPT/CVT: the angle between the odontoid process tangent through cv2ip and the tangent to the odontoid process through cv4ip. NSL/VER: the angle between the nasion-sella line and true vertical. OPT/HOR: the angle between the line OPT and true horizontal. CVT/HOR: the angle between the line CVT and true horizontal. NSL/ML (cranium basis inclination related to the mandible). NSL/CVT (cranium inclination in relation to $\mathrm{C2}$ and in relation to the cervical spine). Transversal roll and sagittal pitch are the units used in the inclinometer studies. RPE: rapid palatal expansion. RME: rapid maxillary expansion.

\begin{tabular}{|c|c|c|c|c|c|c|}
\hline Study & Sample Size & Treatment Design & Age & Sex & Type of Treatment & Criteria of Study \\
\hline $\begin{array}{c}\text { Yagci et al. } \\
\text { [12] }\end{array}$ & 38 & $\begin{array}{c}23 \text { patients (test) } \\
15 \text { patients (control) }\end{array}$ & $\begin{array}{c}10.1 \pm 1.1 \\
9.7 \pm 1.4\end{array}$ & $\begin{array}{l}20 \mathrm{~F} \\
18 \mathrm{M}\end{array}$ & RME & $\begin{array}{l}\text { Transversal roll } \\
\text { Sagittal pitch }\end{array}$ \\
\hline $\begin{array}{c}\text { Tecco et al. } \\
{[13]}\end{array}$ & 45 & $\begin{array}{c}23 \text { patients (test) } \\
22 \text { patients (control) }\end{array}$ & $\begin{array}{l}8-15 \text { years } \\
8.1 \pm 2.0\end{array}$ & $45 \mathrm{~F}$ & RPE & $\begin{array}{l}\text { CVT/EVT } \\
\text { OPT/Ver } \\
\text { CVT/Ver } \\
\text { EVT/Ver } \\
\text { SN/OPT } \\
\mathrm{SN} / \mathrm{CVT} \\
\mathrm{PP} / \mathrm{OPT} \\
\mathrm{PP} / \mathrm{CVT} \\
\mathrm{MP} / \mathrm{OPT} \\
\mathrm{MP} / \mathrm{CVT}\end{array}$ \\
\hline $\begin{array}{c}\text { Mc Guinness et al. } \\
{[14]}\end{array}$ & 79 & $\begin{array}{c}43 \text { patients (test) } \\
36 \text { patients (control) }\end{array}$ & $10-16$ years & $\begin{array}{c}49 \mathrm{~F} \\
30 \mathrm{M}\end{array}$ & RME & $\begin{array}{l}\text { OPT/NSL } \\
\text { CVT/NSL } \\
\text { OPT/CVT } \\
\text { NSL/VER } \\
\text { OPT/HOR } \\
\text { CVT/HOR }\end{array}$ \\
\hline $\begin{array}{c}\text { Tecco et al. } \\
{[15]}\end{array}$ & 45 & $\begin{array}{c}23 \text { patients (test) } \\
22 \text { patients (control) }\end{array}$ & $\begin{array}{c}8-15 \text { years } \\
8.1 \pm 2.0\end{array}$ & $45 \mathrm{~F}$ & RPE & $\begin{array}{l}\text { CVT/EVT } \\
\text { OPT/Ver } \\
\text { CVT/Ver } \\
\text { EVT/Ver } \\
\text { SN/OPT } \\
\text { SN/CVT } \\
\mathrm{PP} / \mathrm{OPT} \\
\mathrm{PP} / \mathrm{CVT} \\
\mathrm{MP} / \mathrm{OPT} \\
\mathrm{MP} / \mathrm{CVT}\end{array}$ \\
\hline
\end{tabular}


Table 2. contd...

\begin{tabular}{|c|c|c|c|c|c|c|}
\hline Study & Sample Size & Treatment Design & Age & Sex & Type of Treatment & Criteria of Study \\
\hline $\begin{array}{c}\text { Yagci et al. } \\
{[16]}\end{array}$ & 45 & $\begin{array}{c}15 \text { patients (test } 1 \text { ) } \\
15 \text { patients (test } 2 \text { ) } \\
15 \text { patients } \\
\text { (control) }\end{array}$ & $\begin{array}{l}9.6 \pm 1.3 \\
9.5 \pm 1.5 \\
9.8 \pm 1.6\end{array}$ & $\begin{array}{l}22 \mathrm{~F} \\
23 \mathrm{M}\end{array}$ & $\begin{array}{c}\text { RPE; } \\
\text { Conventional face- } \\
\text { mask therapy; } \\
\text { Modified facemask } \\
\text { therapy }\end{array}$ & $\begin{array}{l}\text { Transversal roll } \\
\text { Sagittal pitch }\end{array}$ \\
\hline $\begin{array}{c}\text { Kilinc et al. } \\
\text { [17] }\end{array}$ & 35 & $\begin{array}{c}18 \text { patients (test) } \\
17 \text { patients } \\
\text { (control) }\end{array}$ & $\begin{array}{l}10.9 \\
10.9\end{array}$ & $\begin{array}{l}20 \mathrm{~F} \\
15 \mathrm{M}\end{array}$ & RPE & $\begin{array}{l}\text { NSL/ML } \\
\text { NSL/CVT }\end{array}$ \\
\hline
\end{tabular}

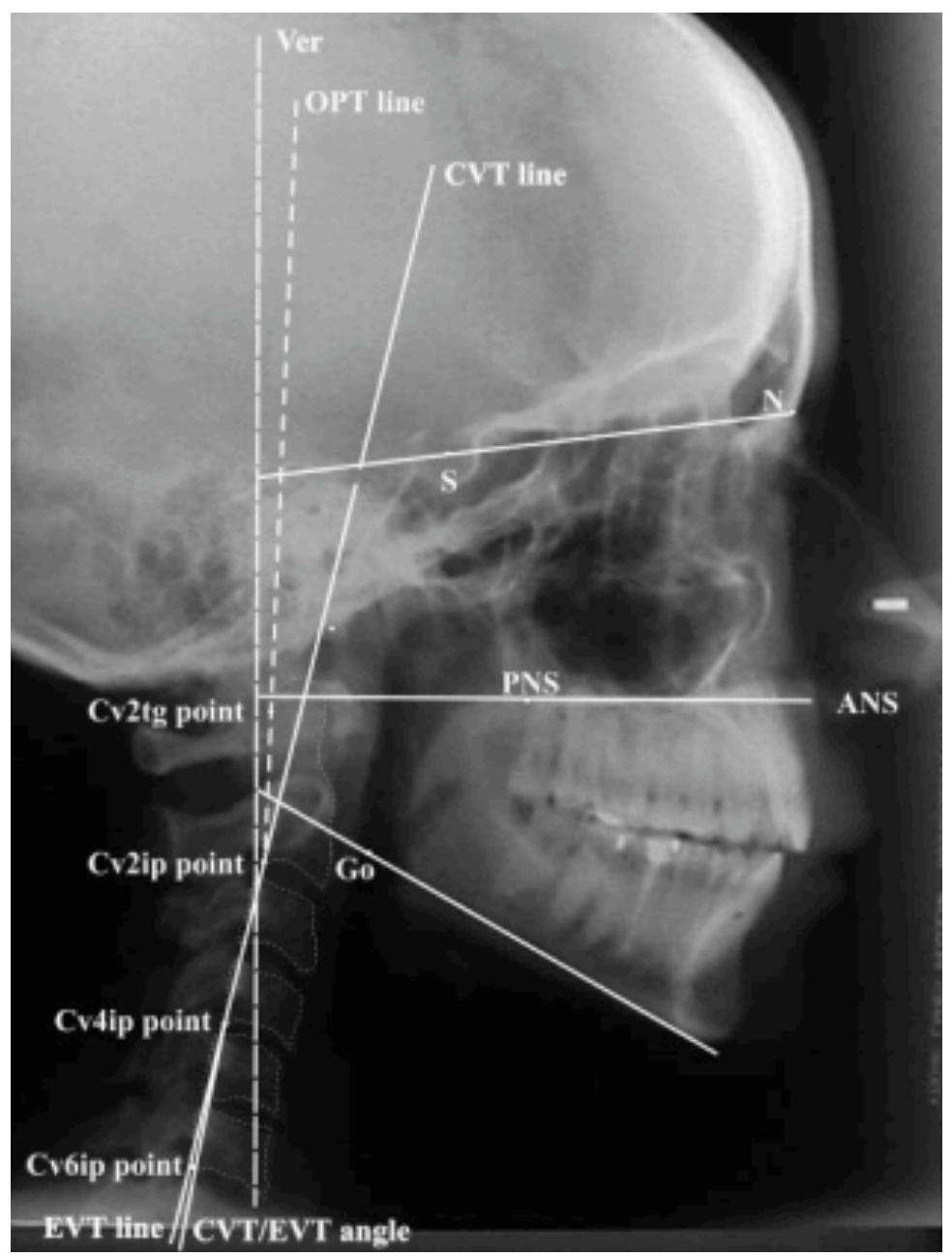

Fig. (1). Cephalometric indexes used in two articles [13, 15]. CVT: the upper part of the cervical spine. A line through cv2tg and cv4ip. EVT: the lower part of the cervical spine. A line through cv4ip and cv6ip. OPT: odontoid line. A line through cv2tg and cv2ip. SN: anterior cranial base. A line through point S and point N. PP: palatal plane. A line through posterior nasal spine (pns) and anterior nasal spine (ans). MP: mandibular plane. A tangent line to the inferior border of the mandible. Variables: CVT/EVT: cervical lordosis angle. The downward opening angle between the CVT and EVT line. OPT/Ver: odontoid angle. The downward opening angle between the OPT and true vertical lines. EVT/Ver: lower cervical column angle. The downward opening angle between the EVT and true vertical lines. CVT/ Ver: upper cervical column angle. The downward opening angle between the CVT and true vertical lines. SN/Ver: the downward opening angle between the SN and true vertical lines. PP/Ver: the downward opening angle between the palatal and true vertical lines. MP/Ver: the downward opening angle between the mandibular and true vertical lines. SN/OPT: the downward opening angle between OPT and SN lines. SN/CVT: the downward opening angle between CVT and SN lines. PP/OPT: the downward opening angle between OPT and palatal lines. PP/CVT: the downward opening angle between CVT and palatal lines. MP/OPT: the downward opening angle between OPT and mandibular lines. MP/CVT: the downward opening angle between CVT and mandibular lines. 


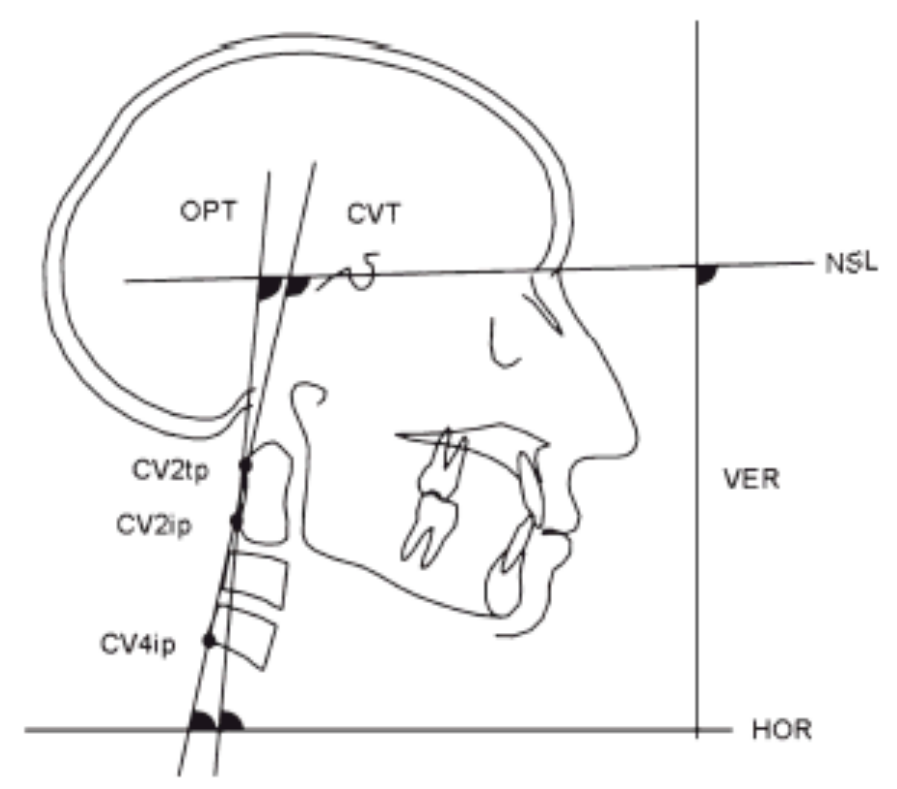

Fig. (2). Craniocervical angulations used in the study of Mc Guinness at al. [14] OPT/NSL: the angle formed by the line tangent to the odontoid process (CV2tp) through cv2ip (the most inferior and posterior point on the corpus of the second cervical vertebra), and the nasion-sella line. CVT/NSL: the angle formed by the line tangent to the odontoid process through cv4ip (the most inferior and posterior point on the fourth cervical vertebra), and the nasion-sella line. OPT/CVT: the angle between the odontoid process tangent through cv2ip and the tangent to the odontoid process through cv4ip. NSL/VER: the angle between the nasion-sella line and true vertical. OPT/HOR: the angle between the line OPT and true horizontal. CVT/HOR: the angle between the line CVT and true horizontal.

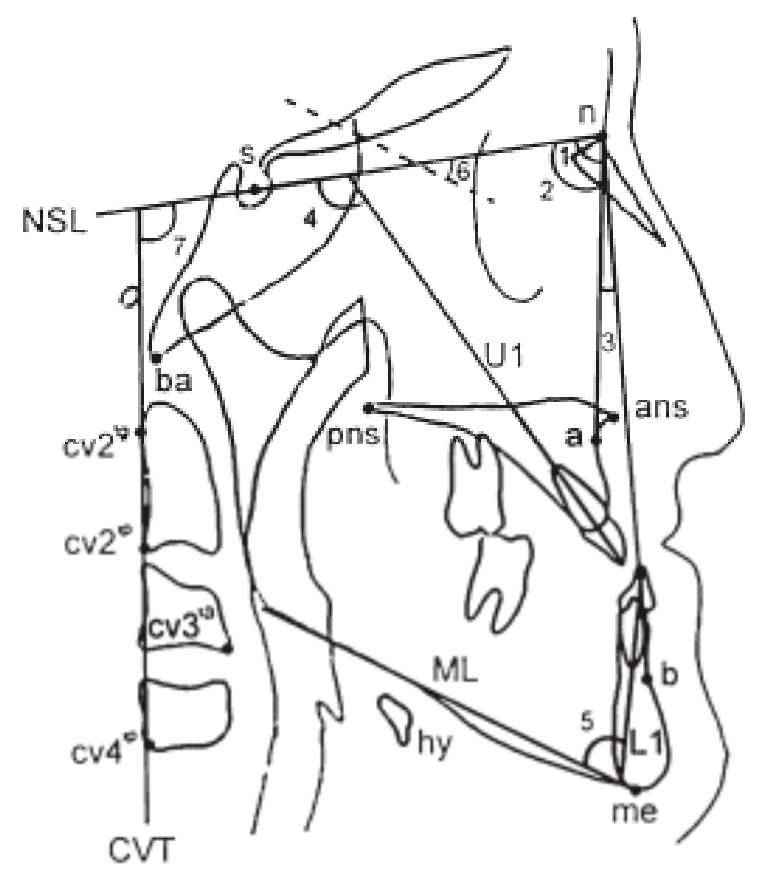

Fig. (3). Craniocervical angulations used in the study of Kilinc et al [17]. Reference points and angular measurements. Reference points (Linder-Aronson, 1970): Hyoid (hy), the most postero-superior point on the body of the second cervical vertebra (cv2); cv2 ${ }^{\text {tg }}$, the most postero-inferior point on the body of cv2; $\mathrm{cv} 2^{\mathrm{ip}}$, the most postero-inferior point on the body of cv2; cv $4^{\text {ip }}$, the most antero-inferior point on the body of the fourth cervical vertebra $\left(\mathrm{cv} 4^{\mathrm{ia}}\right)$; $\mathrm{ad}^{2}$, the intersection between a line from posterior nasal spine (pns) to the midpoint of a line joining basion (ba) and sella (s) and the posterior contour of the adenoid soft tissue shadow; $\mathrm{ad}^{1}$, the intersection between a line from pns to ba and the posterior contour of the adenoid soft tissue shadow; APW, the anterior pharyngeal wall along the line intersecting cv $2^{\text {ia }}$ and hy; PPW, the posterior pharyngeal wall along the line intersecting $\mathrm{cv} 2^{\text {ia }}$ and hy; $\mathrm{APW}^{\prime}$, the anterior pharyngeal wall along the line intersecting cv ${ }^{\text {ia }}$ and hy; $\mathrm{PPW}^{\prime}$, the posterior pharyngeal wall along the line intersecting cv4 ${ }^{\text {ia }}$ and hy. Angular measurements: 1-SNA, 2-SNB, 3-ANB, 4-U1 to NSL, 5-L1 to ML, 6-NSL/ML, 7-NSL/CVT; NSL, nasion sella line; ML, mandibular plane; NSL-CVT, the angle between line NSL and the line from $\mathrm{cv} 4^{\mathrm{ip}}$ to $\mathrm{cv} 2^{\mathrm{ip}}$ (cervical vertebra tangent). 
curvature angle in children and adolescents with nasal obstruction using the RPE appliance. Twenty-three female patients aged 8 to 15 years were included in the test group, and 22 age- and sex-matched patients were included in the control group. The protocol was: 4 turns on the first day followed by 2 turns per day until the required expansion was achieved. Lateral cephalograms were used to measure the craniocervical angulation and the nasopharyngeal airway adequacy. The long-term evaluation was 12 months. One year after expansion, nasopharyngeal airway adequacy, measured by Pm-Ad 2, increased by $5.3 \mathrm{~mm}$, the angles $\mathrm{SN} / \mathrm{OPT}$ decreased by 5.1 , the angle PP/OPT decreased by 4.36, and the angle MP/OPT decreased by 5.12 [8]. All of these results were statistically significant. These findings indicated a reduction in head elevation. The correlation coefficients indicated a significant correlation between nasopharyngeal airway size and craniocervical angulation. The authors concluded that an improvement of nasopharyngeal airway adequacy as a result of RPE is associated with a decrease in craniocervical angles and an increase in cervical curvature angle and head flexion $(\mathrm{p}<0.05)$.

Mc Guinness et al. [14] studied the long-term effects of RME with a bonded-type appliance, in craniocervical angulation. The treatment protocol was of 2 turns per day for an average of 3 weeks. This study used lateral cephalograms to measure the craniocervical angulations. Patients from 10 to 16 years of age were included: 39 subjects in the test group and 36 patients in the control group. The long-term evaluation was completed 12 months later. One year after expansion of the palate, the angle NSL/VER decreased by 3.14 degrees, and the angles OPT/HOR by 2.13 degrees and CVT/HOR by 2.55 degrees [8]. All these results were statistically significant. These findings indicate a reduction in head elevation and, according to the authors, is consistent with improved nasal respiration.

Tecco et al. [15] studied the changes in head posture after rapid maxillary expansion in 45 mouth breathing girls (8-15 years of age). The treatment group comprised 23 girls treated with RME appliance with a protocol of 4 turns on the first day followed by 2 turns per day until the required expansion was achieved, the control group comprised 22 girls followed about 8 months before beginning therapy and became untreated controls. Dental casts and lateral skull radiographs exposed in natural head position were obtained at the first visit and 6 months later for all subjects. In the girls under active treatment there was a statistically significant increase of pm-Ad $2(p<0001)$, a significant increase of the cervical lordosis angle $(p<0001)$, a flexion of the head $(p<0001)$, and a decrease in the craniocervical angulation $(p<0001)$. No significant changes were seen in the control group. RME is capable of increasing nasopharyngeal airway adequacy in girls, and consequently these results confirm an increase of head flexion.

Yagci et al. [16] valuated the effects of varying force directions on the dynamic measurement of natural head position and orofacial airway dimensions of Class III patients (30 patients divided in two groups, conventional and modified facemask therapies) during maxillary orthopedic protraction compared with an untreated control group (15 patients). The mean age was about 10 years. Natural head position meas- urements, obtained trought the use of an inclinometer and cephalometric records were obtained from all subjects before and after treatment or the control period (approximately 1 year). Both treatment groups showed statistically significant changes in the sagittal measurements of natural head position and upper pharynx. In the control group, the only statistically significant change was an increased upper pharynx measurement $(p=020)$. Statistically significant natural head position differences were found in the conventional $\left(6.4^{\circ}\right.$ flexion) and the modified (5.7 $7^{\circ}$ flexion $)$ facemask groups when compared with the controls. The modified facemask group also showed significant changes in aerial $(p=.003)$ and total $(\mathrm{p}<.001)$ areas of the airway measurements compared with the control group. No statistically significant differences were observed between the 2 treatment groups. These findings suggest that modified and conventional facemask therapy with expansion have significant cranial flexion effects on the dynamic measurements of natural head position. Additionally, the modified facemask procedure showed significant effects on the orofacial airway dimensions compared with the initial values and the values of the untreated controls.

Kilinc et al. [17] examined the effects of rapid palatal expansion (RPE) and maxillary prolonging headgear therapy in 18 patients with a skeletal Class III malocclusion and on upper airway dimensions compared with 19 patients of the untreated control group. In both groups the mean age was 11 years. Pre- and post-treatment cephalometric lines and angles was analyzed in both groups. The angles analyzed to evaluate the changes in the position of the head have been NSL/ML and NSL/CVT. The results showed that the first angle is increased considerably, as did the second. After treatment, the head was in a more extended position in relation to the cervical vertebrae, as demonstrated by a mean increase of 2.64 degrees in the NL/CVT angle.

\section{DISCUSSION}

This review of literature focused on changes produced by RME on head posture. Because of the great complexity of cranial anatomy and function, several measurement methods have different objectives and can complete each other to assess the real changes in NHP. Although the cephalometric radiographs in the selected studies were taken according to a standardized technique, the different structure superimpositions and image magnifications did not always allow accurate quantifications of the changes. However, also important was the lack of use of blinded examinations of the subjects and control. Many errors may result from the incorrect use of the head positioners for the cephalometric technique. These factors could influence the measured variable of head posture $[13-15,17]$. Tecco et al. analyzed only female subjects in these standardized studies because female have a particular vertebral curvature, with a partial reverse curvature more easily visible. Altough these findings confirm this hypothesis, it is very uncertain if these changes in posture head are clinically relevant $[13,15]$. Also, analyzing mouth breathers girls they excluded "patients with open bite" as well as those with "nasal allergic conditions or airway obstructions due to adenoids". However, they did not report, how they gathered this information. In addition, these conditions are very often 
present in patients presenting with the need for maxillary expansion. A possible hypothesis for the role of RME in postural changes may be that the increase in palatal width results in enlargement of the pharyngeal airway space, improvement in respiratory function and flexion of the head on the cervical column, with an increase in the cervical curvature angle and a decrease in craniocervical angulation. In this mechanism, the muscular-neural network could play an important role [14]. There is also in the recent literature, an important case report in which the authors try to hypothesized that the changes in the palatal width obtained with RME appliances can involve other bone structures such as the tongue's muscles and the suboccipital muscles. These structures are functionally bound and cooperate to manage the head and neck position, [18-23]. Solow et al. [19] and, Solow and Greve [20] observed a significant relationship between nasal airway adequacy and changes in posture head. Infact, the common interpretation of these results is that the mouth breathers have an extension of the head and this could an important compensation for nasal airway inadequacy [21]. Mc Guinness et al. [14], used an historical control sample because it was not possible to obtain an untreated control group and exposed them to radiographs for comparisons. The recordings of the study of Kilinc et al. [17] have limitations because were based on two dimensional cephalometric measurements, and not on three dimensional measurements. Finally, the authors in two studies [16, 17] have found changes in the position of the head after the treatment of rapid palatal expansion before to continue the therapy with the advancement of the maxilla (subjects with a skeletal class III malocclusion). The studies that used the inclinometer showed a good reproducibility of recordings over time and a lower mean method error [12,16]. Yagci et al. [16] used the inclinometer and the recordings were accurate. However, the measurements should be reproducible over long periods, and the accuracy of the recordings should not depend entirely on the operator's skill. In this study both females and males were studied, but the sex differences were not determined. The increase in flexion of the head was found in both treatment groups [16]. Yagci et al. in the other study analyzed [12] supported the hypothesis in which there is no statistically significant difference between RME and changes in natural head position in the test group and in the control group. The results suggested that dynamic measurement of NHP recorded by an inclinometer apparatus, which is most probably dictated by maintenance of visual axis, is not significantly affected by RME. However, this does not necessarily mean that the craniocervical relation is not affected by RME. Although the few studies that used an alternative technique did not consistently find differences between subjects and controls $[12,16]$. By analysis of the literature it appears to be strongly evident that of the different operational protocols were used in the different studies (Tab.II), both as regards the use of equipment, the methods of analysis (for example inclinometer or cephalometric angles), and the choice (sex, age) of the components of the two groups (test group and control group).

\section{CONCLUSION}

From the analysis of the recent scientific literature, it has been noted that RME causes not only dentoalveolar changes, but also craniocervical structure changes [5]. It has been hypothesized that, since the maxillary bones form a half of the nasal cavity's structures, when the midpalatal suture is open, the nasal cavity's lateral walls are also displaced apart, and its volume increases, and upper airway resistance decreases over time. Head posture had also been associated with respiratory function, and increased craniocervical angulation was observed as a functional response to facilitate oral breathing to compensate for nasal obstruction $[21,22]$.

In conclusion, this review of literature suggests that the potential relationship between RME and head posture is still unclear. Although it is evident a change in the position of the head after the rapid palatal expansion, it cannot be quantified in an objective way since the few studies reported in the literature did not use the same evaluation method. Cephalometric angles, and different measurement techniques do not allow to quantify properly the actual change. There is a need for further well controlled long-term clinical trials using most precise methods to measure craniocervical anatomy and its function and to look at the data for a longer observation period. There is also a need of Long-term stability results in the Rapid palatal expansion $[2,23]$.

\section{CONFLICT OF INTEREST}

The authors confirm that this article content has no conflicts of interest.

\section{ACKNOWLEDGEMENTS}

Declared none.

\section{REFERENCES}

[1] Angell EH. Treatment of irregularity of the permanent or adult teeth. Dental Cosmos 1860; 1: 540 .

[2] Zuccati G, Casci S, Doldo T, Clauser C. Expansion of maxillary arches with crossbite: a systematic review of RCTs in the last 12 years. Eur J Orthod 2011. [Epub 2011/12/14].

[3] Mundstock KS, Barreto G, Meloti AF, Araujo MA, dos SantosPinto A, Raveli DB. Rapid maxillary expansion with the Hyrax appliance: an occlusal radiographic evaluation study. World J Orthod 2007; 8(3): 277-84.

[4] Franchi L, Baccetti T, Lione R, Fanucci E, Cozza P. Modifications of midpalatal sutural density induced by rapid maxillary expansion: a low-dose computed-tomography evaluation. Am J Orthod Dentofac Orthop 2010; 137(4): 486-8; [discussion 12A-13A]. Epub 2010/04/07.

[5] Haas AJ. The treatment of maxillary deficiency by opening the midpalatal suture. Angle Orthod 1965; 35: 200-17.

[6] Blackwelder WC. Showing a treatment is good because it is not bad: when does "noninferiority" imply effectiveness? Control Clin Trials 2002; 23(1): 52-4.

[7] Di Vece L, Faleri G, Picciotti M, Guido L, Giorgetti R. Does a transverse maxillary deficit affect the cervical vertebrae? a pilot study. Am J Orthod Dentofac Orthop 2010; 137(4): 515-9.

[8] Baratieri C, Alves M Jr., de Souza MM, de Souza Araujo MT, Maia LC. Does rapid maxillary expansion have long-term effects on airway dimensions and breathing? Am J Orthod Dentofac Orthop 2011;140(2):146-56.

[9] Guijarro-Martinez R, Swennen GR. Cone-beam computerized tomography imaging and analysis of the upper airway: a systematic review of the literature. Int J Oral Maxillofac Surg 2011; 40(11):1227-37.

[10] Domann CE, Kau CH, English JD, Xia JJ, Souccar NM, Lee RP. Cone beam computed tomography analysis of dentoalveolar changes immediately after maxillary expansion. Orthodontics 2011;12(3): 202-9. 
[11] Gamble J, Lagravere MO, Major PW, Heo G. New statistical method to analyze three-dimensional landmark configurations obtained with cone-beam CT: basic features and clinical application for rapid maxillary expansion. Korean J Radiol 2012; 13(2): 126-35.

[12] Yagci A, Uysal T, Usumez S, Orhan M. Rapid maxillary expansion effects on dynamic measurement of natural head position. Angle Orthod 2011; 81(5): 850-5.

[13] Tecco S, Caputi S, Festa F. Evaluation of cervical posture following palatal expansion: a 12-month follow-up controlled study. Eur J Orthod 2007; 29(1): 45-51.

[14] McGuinness NJ, McDonald JP. Changes in natural head position observed immediately and one year after rapid maxillary expansion. Eur J Orthod 2006; 28(2): 126-34.

[15] Tecco S, Festa F, Tete S, Longhi V, D'Attilio M. Changes in head posture after rapid maxillary expansion in mouth-breathing girls: a controlled study. Angle Orthod 2005; 75(2): 171-6.

[16] Yagci A, Uysal T, Usumez S, Orhan M. Effects of modified and conventional facemask therapies with expansion on dynamic measurement of natural head position in Class III patients. Am J Orthod Dentofac Orthop 2011; 140(5): e223-31.

[17] Kilinc AS, Arslan SG, Kama JD, Ozer T, Dari O. Effects on the sagittal pharyngeal dimensions of protraction and rapid palatal expansion in Class III malocclusion subjects. Eur J Orthod 2008; 30(1): 61-6.

[18] Monaco A, Tepedino M, Sabetti L, Petrucci A, Sgolastra F. An adolescent treated with rapid maxillary expansion presenting with strabismus: a case report. J Med Case Rep 2013; 7(1): 222.

[19] Solow B, Siersbaek-Nielsen S, Greve E. Airway adequacy, head posture, and craniofacial morphology. Am J Orthod 1984; 86(3): 214-23.

[20] Sorensen H, Solow B, Greve E. Assessment of the nasopharyngeal airway. A rhinomanometric and radiographic study in children with adenoids. Acta Oto-Laryngol 1980; 89(3-4): 227-32.

[21] Ricketts RM. Respiratory obstruction syndrome. Am J Orthod 1968; 54(7): 495-507.

[22] Oliveira De Felippe NL, Da Silveira AC, Viana G, Kusnoto B, Smith B, Evans CA. Relationship between rapid maxillary expansion and nasal cavity size and airway resistance: short- and long-term effects. Am J Orthod Dentofac Orthop 2008; 134(3): 370-82.

[23] Zuccati G, Casci S, Doldo T, Clauser C. Expansion of maxillary arches with crossbite: a systematic review of RCTs in the last 12 years. Eur J Orthod 2013; 35(1): 29-37.

(C) Ortu et al.; Licensee Bentham Open.

This is an open access article licensed under the terms of the Creative Commons Attribution Non-Commercial License (http://creativecommons.org/licenses/by-nc/3.0/) which permits unrestricted, non-commercial use, distribution and reproduction in any medium, provided the work is properly cited. 